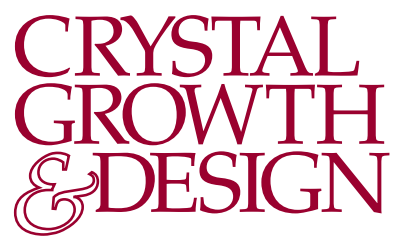

\title{
Crystallizing New Frameworks for Cooperation within Asia
}

7 he second China-India-Singapore (CIS) meeting on 1 Crystal Engineering was held November 19-23, 2012 at the Sun Yat-Sen University, Guangzhou, China. The meeting fosters better cooperation among the neighbors in Asia. The theme for the meeting revolves around the crystal structure and the general principles governing the organization of molecular and modular solids. The focus of the meeting is to generate cooperation and understanding and to foster good nexus in cutting edge research areas in chemistry. A meeting between the scientists of India and China is significant in more ways than one, as there had not been any scientific interactions at the researcher level before. This meeting provided the platform for closer interactions between the researchers in the general areas of inorganic materials and organic solids.

It has been the general belief that the countries Brazil, Russia, India, China, and South Africa (BRICS) would lead the economic charge in the 21st century. The rapid industrialization brought about an increase in the per capita (growth in GDP) in these countries in recent years and lends credence to this belief. Within Asia, Japan is, probably, the most developed both scientifically, as well as economically, followed by South Korea and Singapore. China and India, both huge countries, are catching up with these countries in the development of scientific and industrial growth quickly. The coming together of all these countries from Asia would be expected to form a strong scientific conglomerate in the future.

The economic upturn witnessed in India and China during the last two decades facilitated generous funding from the governments for both the basic and the applied research. ${ }^{1}$ The increased funding from the government sources helped in improving the available infrastructure for research, especially in these two countries. In addition, the funds provided better opportunities for many researchers to fruitfully engage in research. This resulted in a visible increase in the publication of good quality scientific papers from India and China.

It has been the belief that the synergetic efforts in research between India and China would be mutually beneficial for both countries. Singapore provided the initial opportunity toward this synergy by hosting the first trilateral scientific meeting in the area of crystal engineering. The first meeting of this nature, envisaged by Professors G. R. Desiraju (India), J. J. Vittal (Singapore), and Miao Du (China), was hosted by National University, Singapore, during July 31 through August 2, 2010. ${ }^{2}$ The importance of crystal engineering in the design of solids and their properties was focused on in this meeting, which was attended by about 50 participants. This meeting, as hoped, helped in crystallizing new partnerships and understanding among Asian neighbors. The editor-in-chief, Professor Robin Rogers, of one of the leading journals in the area of crystal engineering, Crystal Growth \& Design, stressed the importance and significance of the coming together of scientists from India, China, and Singapore and their role in advancing the sciences in his editorial. ${ }^{3}$

The second meeting on crystal engineering between the three countries was organized in Sun Yat-Sen University,
Guangzhou, China, by Professor X. M. Chen. The meeting had about 75 total participants with 15 scientists from India, 5 from Singapore, and the remaining from China. The smaller group of scientists has the advantage of having closer and more open interactions among the participants. The inclusive nature of the principles of crystal engineering in molecular and modular porous solids was evident during the meeting. A number of metal-organic framework compounds and heterometallic clusters have been prepared employing subtle crystal engineering approaches. In addition, other useful areas that employ crystal engineering principles such as the covalent organic framework solids, organic semiconductors, etc. were also highlighted. The usefulness of $3 \mathrm{D}$ electron diffraction for the structural studies of micrometer-sized particles was demonstrated. The many presentations at the meeting provided important pointers for the design of multifunctional solids though crystal engineering. The richness of the science being practiced in the three countries was evident through the presentations at the meeting.

The importance of meetings of this nature can be measured by the impact it is likely to have by fostering improved scientific cooperation through personal interactions. The discussions among the scientists during the meeting provided encouraging pointers toward this new enthusiasm to foster friendship and mutual cooperation. Efforts of this nature may augment the bilateral cooperation efforts initiated by the governments of China and India. The researcher level interactions created a clear consensus among participants that interdisciplinary meetings of this nature must be promoted.

To further the enthusiasm and interest witnessed during the first two meetings among the scientists from the Asian neighbors, it was decided to hold the third meeting at Bangalore, India, during December 2014. One of the suggestions from the discussions at the second CIS meeting is to engage and encourage intense participation from research students and postdoctoral researchers, as they are the future leaders of science. Though the first two meetings were restricted to the participation of scientists from China, India, and Singapore, it was felt that the other neighbors within Asia may also be encouraged to participate in this third meeting, on invitation. Thus, scientists from Thailand, Malaysia, Indonesia, Vietnam, Mongolia, Cambodia, and South Korea may be invited to attend the third meeting to be held in Bangalore. It is hoped that such an event would augur well for the mutual growth of science as well as provide closer interactions among scientists in this region.

S. Natarajan,* Guest Editor

Solid State and Structural Chemistry Unit, Indian Institute of Science, Bangalore 560 012, India

\section{AUTHOR INFORMATION}

\section{Corresponding Author}

*E-mail: snatarajan@sscu.iisc.ernet.in.

Published: April 3, 2013 


\section{Notes}

Views expressed in this editorial are those of the author and not necessarily the views of the ACS.

\section{REFERENCES}

(1) Arunan, E.; Brakaspathy, R.; Desiraju, G. R; Sivaram, S. Angew. Chem., Int. Ed. 2013, 52, 114-117.

(2) Biradha, K.; Su, C.-Y.; Vittal, J. J. Cryst. Growth Des. 2011, 11, 875-886. Verma, S. Curr. Sci. 2010, 99, 717.

(3) Rogers, R. D. Cryst. Growth Des. 2010, 10, 4671; Cryst. Growth Des. 2011, 11, 1-3. 